\title{
Neuigkeiten vom Buch- und Zeitschriftenmarkt · Articles and Books
}

\section{Verhaltenstherapie}

\section{Buchbesprechungen}

\section{Döpfner, M., Schürmann, S., Frölich, J.}

Therapieprogramm für Kinder mit hyperkinetischem und oppositionellem Problemverhalten - THOP

Weinheim, Psychologie Verlags Union, 1997, 416 S., DM 128,ISBN 3-621-27356-5

Mit dem «Therapieprogramm für Kinder mit hyperkinetischem und oppositionellem Problemverhalten - THOP» haben die Autoren und die Autorin ein neues, auf den Einzelfall zuschneidbares Standardprogramm zur Therapie hyperaktiver und oppositionell-aggressiver Kinder vorgelegt, welches aus 21 Behandlungsbausteinen mit jeweils kindzentrierten und familienzentrierten Interventionen besteht. Zwar werden im Buch keine konkreten Altersangaben gemacht, betrachtet man aber die umfangreichen Therapiematerialien im Anhang, so ist das THOP für Kinder vom Kindergartenalter bis zum Alter von etwa maximal 12 Jahren geeignet. Da hyperaktive und oppositionell-aggressive Kinder in dieser Altersstufe einerseits zu den häufigsten Klienten in Beratungsstellen, freien Praxen, Ambulanzen und anderen Anlaufstellen für Familienprobleme gehören und andererseits als nur schwer therapierbar gelten, ist eine praxisorientierte Hilfe für diese Problematik sicherlich auch längst überfällig.

Viele Therapeutinnen und Therapeuten sehen sich selbst sowohl in Geld- als auch in Zeitnot und daher ist der erste Zugang zum THOP möglicherweise beschwerlich: Die erste Schwelle ist zu überwinden, wenn der stattliche Preis bezahlt werden muß, was in Zeiten knapper Kassen z. B. in Beratungsstellen ja bereits eine nennenswerte Anschaffung darstellt. Wenn die Kaufentscheidung gefällt wurde und das Buch dann vorliegt, erscheint es auf den ersten Blick durch die vielen Hervorhebungen, Checklisten, Materialien und Abbildungen etwas unübersichtlich und wird vielleicht erst einmal wieder aus der Hand gelegt. Aber - und das sei als Ergebnis dieser Besprechung vorweggenommen - ein genauerer Blick in dieses Programm lohnt sich allemal, und bei etwas eingehenderer Beschäftigung damit wird die Struktur des Buches auf einmal klar und einfach.

Im ersten Kapitel werden ausführlich und verständlich die Grundlagen (Symptomatik, Differentialdiagnostik, Genese und Verlauf) der angesprochenen Störungsbilder dargestellt. Auch der aktuelle Stand der diesbezüglichen Therapieforschung wird diskutiert, wobei sowohl medikamentöse als auch psychotherapeutische Ansätze für die Arbeit mit den Kindern, den Eltern und den Familien und Ansätze zur Arbeit mit Kindergärten und Schulen diskutiert werden. Deutlich wird bei dieser Darstellung die Notwendigkeit psychotherapeutischer Behandlung - die allerdings in bestimmten Fällen auch medikamentös unterstützt werden sollte.

In den nächsten beiden Kapiteln werden Diagnostik und Verlaufskontrolle sowie die Therapieplanung ausführlich beschrieben. Die Hinweise zur Diagnostik sind umfassend, und z. T. werden für die Diagnose hilfreiche Materialien im Anhang beigefügt. Die Hinweise zur Verlaufskontrolle sind ebenfalls klar und gut nachvollziehbar.

Bevor im Hauptteil des Buches die einzelnen Therapiebausteine ausführlich dargestellt werden, werden grundsätzliche Hinweise zur Durchführung des THOP ausgesprochen übersichtlich gegeben. Neben der Schilderung der allgemeinen Sitzungsstruktur sind die Angaben zur individuellen Zusammenstellung der Therapie in Abhängigkeit von den diagnostischen Ergebnissen äußerst hilfreich. Tatsächlich muß nicht mit jeder Familie jeder Therapiebaustein durchexerziert werden, sondern die einzelnen Elemente des Programmes werden einzelfallorientiert individuell ausgewählt. Schließlich werden noch Probleme des Beziehungsaufbaus, allgemein schwierige Therapiesituationen und Bedingungen von Widerstand und Mißerfolg dargestellt.

Die Durchführungsanleitungen für die einzelnen Therapiesitzungen (Kapitel 5) sind vorbildlich gelungen. Jedes einzelne Therapiemodul wird nach einer kurzen Übersicht hinsichtlich der notwendigen Vorbereitungen, des Sitzungsablaufes und möglicherweise bei der Durchführung dieses Therapiebausteines auftretender problematischer Situationen so ausführlich und gut strukturiert beschrieben, daß man - eine verhaltenstherapeutische Grundausbildung vorausgesetzt - eigentlich nichts falsch machen kann.

Der Zusammenarbeit mit Kindergärten und Schulen wird ein eigenes Kapitel gewidmet, in dem erfreulicherweise nicht nur die konkreten operanten Maßnahmen beschrieben werden, die dort gegebenenfalls durchgeführt werden sollten, sondern auch ebenso ausführlich Hinweise zur Kontaktaufnahme und zum Beziehungsaufbau mit den in diesen Institutionen Arbeitenden und zur allgemeinen Förderung positiver Interaktionen zwischen Erziehern/Erzieherinnen oder Lehrern/Lehrerinnen und dem Kind gegeben werden.

Nach den ebenfalls lesenswerten Fallbeispielen (Kapitel 7) folgt noch eine Diskussion der Möglichkeiten und Grenzen des THOP. Die Erfahrung mit mehr als 100 Familien, mit denen das Programm unter wissenschaftlicher Begleitung durchgeführt wurde, fassen die Autoren und die Autorin dort zusammen. Sie halten fest, daß das Programm für Therapeutinnen und Therapeuten leicht erlernbar ist, daß es bei Klienten und Klientinnen aus allen sozialen Schichten gut anwend- 
bar ist, daß es von den Klienten und Klientinnen gut angenommen wird, daß die Elternzufriedenheit hoch ist und daß es bei der großen Mehrzahl der Fälle zu deutlichen Verbesserungen der Symptomatik gekommen ist - wenngleich vollständige Symptomfreiheit nur in wenigen Fällen erreicht werden konnte. Die Interventionen in Schulen und Kindergärten haben sich als hilfreich erwiesen, sind aber nicht immer implementierbar. Bei starker Symptomatik kann eine begleitende Medikation für den Schulbesuch (nicht aber für die Zeit zu Hause) notwendig sein.

Das Sahnehäubchen dieses Therapieprogrammes stellt sicherlich der annähernd 200 Seiten umfassende Anhang mit diagnostischen und therapeutischen Materialien dar. Hier finden sich Interviewleitfäden, Checklisten, Beobachtungsbögen, Fragebögen, Visualisierungen, Hausaufgabenblätter, Arbeitsblätter für die Eltern und 15 «Wackelpeter- und TrotzkopfGeschichten» für die Arbeit mit den Kindern. Alle Materialien sind optisch ansprechend und (als Kopie) sofort in der praktischen Arbeit einsetzbar.

Allein die zahlreichen Leitfäden für die Eltern, die sich in gut verständlicher Sprache und klar strukturiert mit den verschiedensten Themen beschäftigen (z. B. «Betrachten Sie Ihr Kind von der positiven Seite!»; «Wie man wirkungsvolle Aufforderungen gibt!»; «Wenn Lob alleine nicht ausreicht: Der Punkte-Plan»; «Wie Sie Probleme bei den Hausaufgaben lösen können»; ...), könnten für sich als hilfreiche Materialien bei der Elternarbeit in der Kinderpsychotherapie publiziert werden.

Nach so viel Lob wären wohl auch ein paar kritische Anmerkungen notwendig, doch dafür bietet das Buch wenig Angriffsfläche. Kleinere Verbesserungen wären an einigen Stellen möglich, z. B. fehlt bei den Beschreibungen der einzelnen Therapiesitzungen jeweils der konkrete Hinweis, auf welchen Seiten des Anhangs die dazugehörigen Materialien zu finden sind, was das Auffinden der Materialien etwas erleichtern würde. Eine etwas zweifelhafte Stelle findet sich, wenn bezüglich der Neigung mancher Eltern zu körperlichen Strafen für meinen Geschmack nicht deutlich genug gesagt wird, daß der Therapeut bzw. die Therapeutin diese Art der Bestrafung im Gespräch mit den Eltern ausdrücklich - auch wenn es sich nur um «Klapse» als Erziehungsmittel handelt - mißbilligen sollte. Schließlich muß auch noch kritisch erwähnt werden, daß die Ergebnisse der wissenschaftlichen Überprüfung des Programmes nicht im Detail - und somit nachprüfbar - mitgeteilt werden, da die statistischen Analysen nach Angaben der Autoren und der Autorin noch nicht abgeschlossen sind. Zwar wäre das Buch dann noch umfangreicher geworden, aber für die Leser und Leserinnen nachvollziehbare Darstellungen der Forschungsergebnisse mitzuliefern sollte meiner Meinung nach eigentlich zum Standard bei der Darstellung eines neuen Therapieprogrammes gehören.

Dennoch überzeugt das Therapieprogramm insgesamt vollständig:

- Es ist gut strukturiert und (vielleicht erst auf den zweiten
Blick) ansprechend gestaltet. Die die behandelten Störungsbilder betreffenden wissenschaftlichen Grundlagen werden ausführlich und verständlich dargestellt.

- Das Therapieprogramm wurde entsprechend den aktuellen Forschungsergebnissen aufgebaut, und seine Anwendung wurde wissenschaftlich überprüft.

- Die Durchführung des gesamten Therapieprogramms und jedes einzelnen Bausteines wird detailliert geschildert und ist daher problemlos erlernbar.

- Die Therapiedurchführung wird erleichtert durch eine Fülle optisch und inhaltlich überzeugender Materialien.

In der Praxis wird sich der zunächst hohe Anschaffungspreis schnell bezahlt machen, und zwar dadurch, daß ein wirksames Therapieprogramm durch die beigefügten Materialien, die nicht erst mühsam selbst gestaltet werden müssen, nach kurzer Einarbeitungszeit effektiv einsetzbar ist. Darüber hinaus sind einzelne Materialien auch störungsübergreifend bei der Arbeit an anderen Störungsbildern einsetzbar, etwa einzelne Eltern-Leitfäden oder einige mit der Familie zu erarbeitende Störungs- und Eigenschaftenlisten. Dem THOP ist daher eine weite Verbreitung uneingeschränkt zu wünschen.

M. Borg-Laufs, Essen

Hamm, A.

Furcht und Phobien: Psychophysiologische Grundlagen und klinische Anwendungen

Göttingen, Hogrefe, 192 S., DM 49,80

ISBN 3-8017-0794-6

Um die Besprechung des obengenannten Werkes in einen angemessenen Rahmen zu setzen, erscheint es mir sinnvoll, zunächst Auskunft darüber zu geben, aus welchem Blickwinkel das Buch gelesen wurde. Dieses Vorgehen mag etwas unüblich erscheinen, soll aber im folgenden erklärt werden. Die Betrachtung des bereits Emotionen hervorrufenden Umschlagbildes (der Kopf der «Medusa» von Michelangelo) und die allgemein gehaltene Überschrift «Furcht und Phobien» erweckt zunächst den Eindruck, ein an der Praxis orientiertes Buch vorliegen zu haben. Dieser erste Eindruck wird zudem durch die Verlagswerbung unterstützt, die auf der Rückseite der «Zeitschrift für Klinische Psychologie» (Band 26, 4, 1997) «Furcht und Phobien» gleichzeitig mit einem Buch über Kurzzeitbehandlung von Panikstörung und Agoraphobie und zwei Bänden der Enzyklopädie der Psychologie, Serie «Klinische Psychologie» von Hahlweg und Ehlers als Neuerscheinung ankündigt. In der dazugehörigen Kurzinformation über den Buchinhalt wird nicht verschwiegen, daß es sich hierbei um eine Arbeit im Bereich der psychophysiologischen Grundlagenforschung handelt (entsprechend des auf der dritten Buchseite zu findenden Untertitels). Der Hinweis aber, daß hiermit ein Leitfaden für das praktische Handeln von Psychotherapeuten vorliegt, läßt denselben in seiner durch die bereits genannten Hinweisreize getriggerten, selektiven Wahr- 
nehmung leicht übersehen, daß von einem theoretischen Leitfaden gesprochen wurde.

Aufgrund dieser Einstellung überrascht dann die Kürze des Kapitels über die Definition von Phobien und die relative Unverbundenheit der Darstellung der Klassifikation und Nosologie phobischer Störungen gegenüber der sehr breit angelegten Darstellung wissenschaftlicher Experimente zur psychophysiologischen Erfassung der an der phobischen Reaktion beteiligten subkortikalen und kortikalen Prozesse. Im abschließenden Kapitel über die Behandlung von Phobikern wird dem therapeutisch orientierten Leser deutlich, daß es sich hierbei nicht um die Entwicklung neuer Verfahren auf der Grundlage wissenschaftlicher Untersuchungen handelt, sondern daß es dem Autor gelungen ist, durch die psychophysiologischen Untersuchungen die bisherige verhaltenstherapeutische Vorgehensweise zu bestätigen. Dabei belegt Hamm in eindrücklicher Weise, daß es sich bei systematischer Desensibilisierung bzw. der Expositionsbehandlung nicht um eine reine Veränderung von Stimulus-Response-Verbindungen handelt, sondern daß «das Verlernen von Furcht vornehmlich durch kortikale Strukturen also über kognitive Prozesse gesteuert wird» (S. 155). Somit konnte auch die «kognitive Wende» in der Verhaltenstherapie neuropsychologisch bestätigt werden.

Spätestens zu diesem Zeitpunkt wird klar, daß man dem Inhalt des vorliegenden Buches mit der oben skizzierten Einstellung nicht gerecht werden kann. Das Buch richtet sich primär an wissenschaftlich orientierte Psychophysiologen und vermittelt auf der Grundlage einer sehr breit angelegten Literaturbasis einen umfassenden Überblick über die Entwicklung von Forschungsparadigmen und experimentellen Untersuchungsmethoden.

Die detaillierte Darstellung verschiedener Forschungsergebnisse zu neuronalen Zusammenhängen erscheint als prinzipiell interessant und bereichernd, birgt aber für den im Bereich der Neurologie ungeschulten Leser die Gefahr der Überforderung mentaler Kapazitäten, zumal die Informationen sehr dicht und in entsprechender Fachsprache präsentiert werden, was sicherlich für den mit der Materie vertrauten Spezialisten unproblematisch ist. Ein daher sehr positiv zu bewertender Punkt ist, daß jedes Kapitel mit einer kurzen Zusammenfassung endet. Die erste Zusammenfassung erscheint jedoch erst nach 52 Seiten und vermittelt bei einer Länge von einer halben Seite den Eindruck, daß möglicherweise einige Informationen fehlen.

Aufgrund der großen Zahl der referierten Ergebnisse aus Einzeluntersuchungen an Tieren und Menschen fällt es schwer, den Inhalt des Buches in kurzer Form wiederzugeben. Die von Hamm bereits gelieferten Zusammenfassungen sind dabei zunächst hilfreich, spätestens ab den Kapiteln, in denen über Ätiologie und Behandlung gesprochen wird, handelt es sich dabei jedoch eher um Schlußfolgerungen, die so allgemein sind, daß sie nicht weit über das bereits bekannte therapierelevante Wissen hinausgehen. Im folgenden wird trotz- dem ein Versuch gemacht, die wichtigsten Punkte herauszugreifen.

Nach der Darstellung der Entwicklung von Ordnungsschemata führt Hamm das basale Motivationssystem ein, das nach Lang neben der Valenz des Affektes auch die Richtung bzw. die Intensität als strategische Dimensionen enthält. Anhand von Ergebnissen aus neurowissenschaftlichen Arbeiten erklärt er dessen Wirkung durch die Darstellung von neuronalen Schaltkreisen und arbeitet die Funktion der Amygdala als «zentrale neuronale Struktur für die Integration und Regulierung aversiver Reaktionsprogramme» (S. 39) heraus. Danach führt er das Paradigma der Schreckreaktion ein, durch welches eine Potenzierung der Abläufe des aversiven Reaktionssystems bewirkt werden kann. Das Paradigma der Furchtinduzierten Potenzierung der Schreckreaktion steht dann im Mittelpunkt der weiteren Darstellungen.

Nach dem Kapitel über die theoretischen Grundlagen wird ein Schwerpunkt auf die Symptomatologie verschiedener Phobien gelegt; hier wird auf die unterschiedlichen Formen und die Situationsspezifität von Vermeidungsverhalten eingegangen. Bezüglich der verschiedenen Phobien werden objektspezifische psychophysiologische Reaktionsmuster dargestellt. Diese Beschreibungen führen dann zu der Vermittlung eines Modells, in dem furchtrelevante Informationen in einem neuronalen Netzwerk gespeichert sind, das wiederum in assoziativer Verknüpfung zu einem subkortikal repräsentierten Aversions-Abwehrsystem steht. Die Aktivierung dieses Systems solle dann zu einer Bahnung protektiver Reflexe, wie dem Lidschlagreflex der Schreckreaktion, führen. Hamm belegt anhand von experimentellen Untersuchungsergebnissen, daß die Furcht-induzierte Potenzierung der Schreckreaktion bei allen Phobikern zu beobachten ist. Die Auswahl der im weiteren präsentierten Untersuchungen hat das Ziel, dieses Modell zu bestätigen.

Im Kapitel über die Ätiologie der Phobien werden Untersuchungen zum Einfluß genetischer Faktoren und von kognitiven und emotionalen Lernprozessen behandelt. Hierbei stellt Hamm eigene Untersuchungsergebnisse zur VerbundreizKonditionierung oder zur Furcht-induzierten Potenzierung der Schreckreaktion vor, wobei sowohl die Aversivität des unkonditionierten Reizes als auch der Verlauf der konditionierten Veränderungen untersucht wurden. Interessant sind dabei die Befunde, daß die Schreckreaktion in differenzierterer Weise beeinflußt wird als das elektrodermale System. Daß die Vielzahl an Faktoren, die bei der Entstehung von Phobien eine Rolle spielen, auch in der Furchtbehandlung zu berücksichtigen sind, zeigt erneut das Kapitel über die psychophysiologischen Prozesse im Verlauf von Systematischer Desensibilisierung, Exposition und bei der Pharmakotherapie. Die verhaltenstherapeutische Behandlung von Phobien unter Berücksichtigung emotionaler und kognitiver Faktoren in Verbindung mit der Expositionsbehandlung wird durch diese Darstellungen bestätigt.

Die kurze Inhaltsangabe soll erneut vermitteln, daß die Ziel- 
gruppe weniger die Seite der therapeutisch orientierten als die der wissenschaftlich orientierten Leser darstellt. Diese bekommen zwar eine breite Übersicht über die bisherigen Studien, haben jedoch keine Möglichkeit, diese nach ihrem experimentellen Wert zu beurteilen, da der Aufbau der Untersuchungen nicht oder nur teilweise dargestellt wird und statistische Angaben über die Höhe der referierten Effekte nicht vermittelt werden.

Bezüglich der formalen Gestaltung des Buches kommt für den Leser erschwerend hinzu, daß die Überschriften der einzelnen Kapitel und Unterkapitel kaum vom Text abgesetzt wurden und die Orientierung in dem sehr kompakt gesetzten Text auch nicht durch Hervorhebungen erleichtern. Ähnlich wenig Sorgfalt wurde auch auf die Kontrolle von Orthographie und Trennzeichen verwendet. Zwischen Umschlaggestaltung und Buchinhalt bestehen daher deutliche Diskrepanzen. Trotz aller Kritikpunkte besteht der Verdienst von Hamm darin, daß es ihm gelungen ist, das Wissen über Phobien, das bisher über Beobachtung und therapeutisches Handeln gewonnen wurde, auch auf der Ebene der Physiologie zu belegen.

M. Geibel-Jakobs, Mannheim

\section{Füllgrabe, $U$.}

\section{Kriminalpsychologie}

Frankfurt, Edition Wötzel, 2. Aufl., 1997, 502 S., DM 39,80

ISBN 3-925831-18-5

Der Autor verspricht im Titel seines Buches, eine empirische Kriminalpsychologie darzustellen und macht seine Leser damit neugierig.

Völlig zu recht fordert er eine empirisch wissenschaftliche Überprüfung gängiger Hypothesen zur Kriminalität und die Orientierung an einem empirisch realistischen Weltbild.

Leider löst der Autor die im Titel seines Buches geweckten Erwartungen dann in keiner Weise ein.

Anstelle einer ausgewogenen und integrierenden Darstellung moderner psychologischer, soziologischer und biologischer Theorien findet sich eine willkürlich anmutende Mischung von Fallgeschichten, persönlichen Erfahrungen und der Darstellung einzelner wissenschaftlicher Arbeiten, zu denen der Autor immer auch kommentierend seine eigene Meinung angibt.

Übliche Standards eines wissenschaftlichen Buches werden hier nicht eingehalten, die zitierte Literatur ist alt und berücksichtigt neuere Publikationen kaum, dafür zitiert der Autor dann aber Fernsehsendungen, was ein Licht auf das Niveau dieses Buches werfen mag. So schreibt der Autor auf Seite 105 seines Buches:

«Gemäß einer Fernsehdokumentation (NDR 3, 7.11.1996) hängt Stimmenhören mit einer Gehirnregion im vorderen linken Schläfenlappen zusammen, die Kontrolle über das sprachliche Denken hat.»
Nun wäre ja auch eine populärwissenschaftliche Darstellung dieses Themas durchaus begrüßenswert, wenn diese ausgewogen und kenntnisreich wäre.

Aber auch dieses Vorhaben ist in dem vorliegenden Buch sicher nicht gelungen.

Der Autor geht nämlich von einer Prämisse aus, die eindeutig als überholt zu bezeichnen ist, wenn er auf Seite 64 seines Buches schreibt: «Das kognitive und das statisch biologische Modell sind nicht nur unterschiedlich, sondern miteinander unvereinbar.»

Ganz abgesehen davon, daß auch das biologische Modell keineswegs statisch ist, was der Autor schon in älteren Lehrbüchern der forensischen Psychiatrie hätte nachlesen können - auf solche Zitate verzichtet der Autor aber vollständig und zitiert lieber eigene, völlig verquere Erfahrungen mit der Psychiatrie -, rezipiert eine solche Haltung natürlich in keiner Weise die modernen bio-psycho-sozialen Erklärungsansätze. Auch original psychologische Forschungssätze, etwa der psychophysiologischen Forschung, fallen bei einer solch einseitigen Haltung dann natürlich unter den Tisch.

Da die gesamte Darstellung in diesem Buch von einem solch einseitigen und ideologischen (?) Standpunkt aus vorgenommen wird, ist zu befürchten, daß bei einem mit der Materie nicht vertrauten Leser ein völlig falsches Bild entsteht.

Zusammenfassend muß man leider festhalten, daß dieses Buch für den Wissenschaftler und für den Laien gleichermaßen ungeeignet ist.

H. Dreßing, Mannheim

Thomä, H., Kächele, $H$.

\section{Lehrbuch der psychoanalytischen Therapie. Bd. 1: Grundlagen}

Berlin, Springer, 2. überarb. Aufl., 521 S., DM 98,ISBN 3-540-60391-3

Der gut 500 Seiten umfassende 1. Band des «Lehrbuchs der psychoanalytischen Therapie» stellt eine äußerst inhaltsreiche theoretische Auseinandersetzung mit den Grundlagen der Psychoanalyse anhand der psychoanalytischen Behandlungstechnik dar. Dabei hat die gegenüber der ersten Auflage von 1985 inhaltlich kaum veränderte 2. Auflage von 1996 durch zahlreiche Untergliederungen und optische, auch farblich abgesetzte Hervorhebungen in der Darstellungsform erheblich gewonnen.

Gleich zu Beginn (S. XIX) stellen die Autoren klar, daß sie sich «außerstande (sahen), die psychoanalytische Praxis von einer allgemein akzeptierten Theorie über Entstehung und Verlauf seelischer Erkrankungen abzuleiten». Statt dessen handelt das Werk von den Grundlagen der psychoanalytischen Behandlungstechnik. Psychoanalyse ist das, was Psychoanalytiker tun (S. 50). Die Unterscheidung zwischen den Erkenntnissen, die in einer psychoanalytischen Neurosenlehre systematisiert sind, und ihrer therapeutisch angemessenen 
Anwendung ist den Autoren sehr wichtig (S. 447). So ist die Erkenntnis über die Bedeutung frühkindlicher Traumatisierungen nicht identisch mit einer (von den Autoren entschieden kritisierten) therapeutischen Technik, die dem Erinnern und der rationalen Rekonstruktion der Vergangenheit und entsprechenden Deutungen ein zu großes Gewicht beimaß ( $\mathrm{S}$. 448).

Das Buch folgt einer historisch orientierten Systematik (S. 1), insbesondere entwickeln die Autoren ihre zentralen Überlegungen im Dialog und der Auseinandersetzung mit dem Werk S. Freuds. Eine Vielzahl von Zitaten aus den Arbeiten Freuds und vieler anderer Autoren macht das Buch zu einer Fundgrube der Fachliteratur.

Der hier besprochene erste Band umfaßt folgende Kapitel: 1. Zur Lage der Psychoanalyse. Eine Einführung, 2. Übertragung und Beziehung, 3. Gegenübertragung, 4. Widerstand, 5. Traumdeutung, 6. Das Erstinterview und die Dritten im Bunde, 7. Regeln, 8. Mittel, Wege und Ziele, 9. Der psychoanalytische Prozeß, 10. Zum Verhältnis von Theorie und Praxis.

Die Leitidee des Buches ist der «Beitrag des Analytikers zum therapeutischen Proze $\beta »$ (S. 9). Nichts, was in der Therapie geschieht, stammt nur vom Patienten. Im Mittelpunkt der Betrachtung steht der dyadische Prozeß zweier Subjekte, des Patienten und des Analytikers, und nicht die Beziehung des Subjekts Patient auf das Objekt Analytiker, an dem sich die innerseelische Problematik des Patienten nur abspielt (S. 90 ff.). Die Psychoanalyse muß sich aus den theoretischen Fesseln der Ein-Personen-Psychologie befreien, die die Rolle von Beziehungen auf das innerseelische (ödipale) Konfliktmodell einengt. Die Autoren messen der Rolle der Triebtheorie mit ihrem ökonomischen Prinzip der Triebabfuhr eine wesentliche Bedeutung für die Schwierigkeit zu, Psychoanalyse als Mehrpersonenpsychologie zu verstehen. Unter anderem wird auch die «Gegenfrage-Regel» (daß in der «klassischen» psychoanalytischen Technik jede Frage mit der Gegenfrage beantwortet werden soll, was dem Patienten zu der Frage einfalle) als Überbleibsel der triebökonomischen Therapieauffassung dargestellt: Durch die verweigerte Antwort würde in diesem Modell der Patient rascher zu Einfällen gelangen, die die latente Bedeutung der Frage erklären, wenn der Analytiker die «Befriedigung» der Antwort vorenthält (S. 306). Der Versuch Freuds, das Subjekt Therapeut aus der Betrachtung des Therapieprozesses zu eliminieren, zeigt sich z. B. an der Konzeption der Übertragung als verzerrter Wahrnehmung des Therapeuten, der dem Kranken die Verzerrung und ihre Herkunft aus früher selbst Erlebtem nachweisen müsse. Diese Tendenz zur Einpersonenpsychologie führen die Autoren auf die Bemühung Freuds um Anerkennung der Wissenschaftlichkeit der Psychoanalyse im Sinne einer Orientierung an naturwissenschaftlicher Erkenntnis zurück (S. 92), die dem Therapeuten, der nach Freud ja auch zugleich der psychoanalytische Forscher sein sollte (S. 451), das Element des subjektiven Erlebens nicht zugestehen, vielmehr im Begriff der Gegenübertragung als niederzuhaltendes Störelement aus der
Therapie verbannen wollte. Dagegen setzen die Autoren ihre Auffassung der Psychoanalyse als hermeneutische Technologie. «Es empfiehlt sich, der erklärenden psychoanalytischen Theorie, durch welche die Psychopathologie des Konflikts systematisiert wurde, eine Theorie der Therapie als Systematik der Problemlösung an die Seite zu stellen» (S. 14). Übertragung ist sowenig nur verzerrte Wahrnehmung (als gäbe es den realen Analytiker nicht, nur den «Spiegel» des Patienten), wie Gegenübertragung nur ein überwindbares Störelement auf seiten des Analytikers ist. Dieser selbst ist in der Übertragungsbeziehung real gemeint (und nicht nur die auf ihn übertragenen inneren Objekte des Patienten), und er fühlt und erlebt real. Die Gegenübertragung «entsteht im Analytiker, und er hat sie auch zu verantworten» (S. 118). «Denn wie soll man durch die Kommunikation mit einem bedeutungsvollen Anderen zu sich selbst finden und sich verändern, wenn das Objekt vorgibt, nichts anderes zu sein als das, was man selbst ist?» (S. 118). «Die Anerkennung realistischer Wahrnehmungen (des Patienten) durch den Analytiker ermöglicht es dem Patienten, seelische Akte zu Ende zu bringen und mit dem Subjekt/Objekt eine Übereinstimmung zu erreichen, die eine der wichtigsten Voraussetzungen für die Bildung von Objektkonstanz und Selbstfindung darstellt. Psychische Akte in dieser Weise erledigen zu können, kennzeichnet die genuinen und therapeutisch wirksamen Erfahrungen in der psychoanalytischen Situation» (S. 96). So sehr das «phobische Vermeiden von Gefühlen» (S. 116) kritisiert wird, so entschieden wenden sich die Autoren allerdings auch gegen eine Gegenübertragungsmystik, die alles aus den eigenen Gefühlen zu erkennen glaubt und keiner weiteren Begründung zu bedürfen scheint (S. 114).

Der Leitidee (s. o.) entsprechend ist auch die gleichschwebende Aufmerksamkeit, von Freud der freien Assoziation des Patienten zugeordnet, ein aktives Zuhören, das auswählt und Hypothesen generiert; «... gerade die Selbsttäuschung unbefleckter Wahrnehmung (öffnet) der unwissentlichen, unbewußten und damit schwer zugänglichen Projektion eigener Ideen Tür und Tor» (S. 299).

Mit der entschiedenen Hinwendung zu einem interaktiven Modell der Psychoanalyse verwerfen die Autoren das Triebabfuhrmodell Freuds als unbrauchbar, betonen jedoch zugleich die enorme Bedeutung dieses Modells für die psychoanalytische Metapsychologie und auch für die Praxis (s. o., Gegenfrageregel) (S. 13). Aber: «Man hat ... guten Grund, bei der Kritik des ökonomischen Gesichtspunkts der Libidotheorie das Kind nicht mit dem Bad auszuschütten. ... Die revidierte, soziologisierte Psychoanalyse hat die Neigung, in Adlers Oberflächlichkeit zurückzufallen: sie ersetzt Freuds dynamische, auf das Lustprinzip begründete Theorie durch bloße Ich-Psychologie.» (S. 37). «Um der Intersubjektivität, der Zweipersonenpsychologie, in der psychoanalytischen Technik voll gerecht zu werden, ist es erforderlich, über die traditionellen Objektbeziehungstheorien ebenso hinauszugehen wie über das Modell der Triebabfuhr. Denn alle für den Men- 
schen wesentlichen Objekte bilden sich von Anfang an in einem intersubjektiven Raum, der von vitalen Lustgefühlen (vital pleasures, G. Klein 1969) durchströmt wird, ohne daß diese eng mit dem Triebabfuhrmodell verbunden werden können.» (S. 100).

Die Autoren halten an der zentralen Stellung von Einsicht und Deutung in der psychoanalytischen Technik fest, reflektieren jedoch die Begriffe und ihr Umfeld, z. B. die Rolle des Schweigens, auf dem Hintergrund ihrer Leitidee der Rolle des Psychoanalytikers im therapeutischen Prozeß. Dabei geht es um die Aufgabe des Analytikers, möglichst optimale Bedingungen für eine hilfreiche therapeutische Beziehung bereitzustellen, in der Deutungen hilfreich und Einsichten effektiv werden können (S. 317, 393). Sie heben die große Bedeutung der Anerkennung, die H. Kohut dargestellt hat, für den therapeutischen Proze $\beta$ heraus, in dem «alle Patienten insofern narzistisch labilisiert (sind), als ihr Selbstgefühl - wie bei jedem Menschen - von Bestätigungen abhängig ist» ( $S$. 396). Deutungen werden häufig als Vorwürfe, Kränkungen oder Entwertungen erlebt (S. 377), stereotypes Schweigen führt $\mathrm{zu}$ einer kontraproduktiven Polarisierung von Macht und Ohnmacht zwischen Patient und Analytiker (S. 375). M. Balints Theorie des Neubeginns wird im Zusammenhang der Rolle des Erlebens (als Gegenpol der Einsicht) in der Therapie besprochen. «Der Begriff des Neubeginns erhält seine umfassende Bedeutung in der Theorie der Therapie, wenn man ihn als ein Geschehen im Hier und Jetzt begreift, das der Analytiker ermöglicht» (S. 349). Sowohl Balints Theorie von Neubeginn und Regression als auch Kohuts Theorie des Narzißmus werden als behandlungstechnisch wesentliche Beiträge gewertet, wobei ihre behandlungstechnische Bedeutung getreu der Unterscheidung der Autoren (s. o.) nicht identisch ist mit ihrem Wahrheitsanspruch bezüglich der Ätiologie psychischer Störungen.

Um die therapeutische Veränderung in der analytischen Situation besser $\mathrm{zu}$ verstehen, ziehen die Autoren auch lerntheoretische Modelle heran. So betonen sie die Nähe zwischen dem Lernen am Modell und der analytischen Konzeption der Identifikation, der sie eine zentrale Bedeutung für jeden therapeutischen Prozeß zuschreiben (S. 368). Darüber hinaus zeigen sie auf, wie sich Begriffe wie Generalisation und Diskrimination sowie Assimilation und Akkomodation kognitiver Schemata auf psychoanalytische Konzepte der Übertragung und des Durcharbeitens, insbesondere im Hinblick auf eine Ermöglichung therapeutischer Veränderung unter Berücksichtigung der Angsttoleranz des Patienten anwenden lassen.

Das Kapitel über den Widerstand habe ich bisher nicht berührt.

Zunächst wird betont, daß Widerstandsphänomene im Rahmen einer primär von Hilfesuche geleiteten Arzt-Patient-Beziehung sekundär entstandene Folgen von Beunruhigungen sind, die freilich dann zu Störungen der Therapie führen. Patienten suchen zwar im Bereich ihres Leidens eine Verände- rung, möchten aber die in ihnen gebundenen zwischenmenschlichen Konflikte möglichst unberührt lassen (S. 139). Dabei betonen die Autoren die adaptive, schutzgebende und beziehungsregulierende Funktion von Widerstand und heben aus der schillernden Bedeutungsvielfalt des Begriffs (S. 143) den Widerstand gegen das Bewußtwerden der Übertragung und den Identitätswiderstand nach Erikson heraus (S. 143, 131). Letzterer betont das übergeordnete Regulationsprinzip der Aufrechterhaltung des psychosozial entstandenen Identitäts- oder Selbstgefühls.

Unter dem Thema Widerstand behandeln die Autoren auch die psychoanalytische Auffassung der Affekte. Sie bemängeln eine einseitige, wieder dem triebökonomischen Modell zuzuschreibende Betonung der Angst (vor Triebstärke) und fordern eine differenzierte Affekttheorie ein. In der Diskussion der negativen therapeutischen Reaktion und der unbewußten Schuldgefühle wird die Nützlichkeit der Konzeption des Loslösungs- und Individuationsprozesses nach M. Mahler für das Verständnis der zunächst paradox anmutenden klinischen Beobachtung negativer therapeutischer Reaktionen auf eine Intensivierung der hoffnungsvoll zugewandten (Übertragungs)beziehung betont (S. $151 \mathrm{f}$.). In diesem Zusammenhang erfolgt auch eine Diskussion von Aggression und Destruktivität, wobei der Triebcharakter von Aggression verneint wird. «... die ungeheure Wirksamkeit, die ständige Sprungbereitschaft von Aggressivität und Destruktivität (ist) nur unter der Voraussetzung ihrer reaktiven Natur ausreichend verständlich» (S. 159). Destruktivität steht in Zusammenhang mit dem «biopsychologischen Regulationsprinzip» der Selbsterhaltung (S. 163). «Behandlungstechnisch ist es wesentlich, die vielen Kränkungen aufzusuchen, die der Patient in der analytischen Situation tatsächlich erlebt und nicht nur durch das Vergrößerungsglas in übertriebener Weise wahrnimmt. Die aufgrund der Regression in der analytischen Situation wiederauflebende kindliche Ohnmacht führt reaktiv zu Allmachtsvorstellungen ... Narzistische Patienten bewegen sich im Teufelskreis unbewußter Rachephantasien ... die negative aggressive Übertragung (hat) auch eine distanzregulierende Funktion». Die «narzistische Wut (verwandelt sich regelhaft) in ... vergleichsweise harmlose Rivalitätsaggression, wenn es gelingt, die Kränkungen in der analytischen Situation auf ihre Wurzeln zurückzuführen» (S. 165).

Weiterhin behandeln die Autoren die psychoanalytische Diagnostik und insbesondere die Indikationsstellung für eine psychoanalytische Behandlung, wobei sie für eine adaptive Modifikation der Technik nach den Bedürfnissen der Patienten plädieren und sich vom Anspruch der normativen Idealtechnik (Eissler) und der Polarisierung zwischen psychoanalytischer Standardtechnik und analytischer Psychotherapie abgrenzen, freilich auch für eine Differenzierung der Therapieformen innerhalb des großen Rahmens analytischer Therapien plädieren (S. 51, 234). Scharf kritisieren sie die Tendenz zu immer längeren Weiterbildungsanalysen (je länger, desto besser), nach deren Modell dann auch therapeutische Analysen 
quasi automatisch, nicht indikationsgebunden zur Verlängerung tendieren. Aspekte der Fremdfinanzierung von Psychotherapie in der Bundesrepublik und generell der Rolle der «Dritten im Bunde» (Angehörige, Krankenkasse) für den Therapieprozeß werden behandelt.

Bezüglich der Traumtheorie rücken die Autoren ab von der Freudschen Auffassung, der Traum sei Wunscherfüllung, und verstehen Träume als Weisen der Selbstdarstellung und Problemlösungssuche; dabei betonen sie jedoch die überragende Bedeutung der Sichtweise, «alle seelischen Erscheinungen, also auch den Traum, als Ausdrucksgeschehen von Wünschen und Bedürfnissen zu betrachten» (S. 193).

Im vorletzten Kapitel stellen die Autoren nach der Diskussion verschiedener Modelle des psychoanalytischen Prozesses ihr eigenes Ulmer Prozeßmodell vor. «Wir betrachten den interaktionell gestalteten Fokus als zentrale Drehscheibe des Prozesses und konzeptualisieren von daher die psychoanalytische Therapie als eine fortgesetzte, zeitlich nicht befristete Fokaltherapie mit wechselndem Fokus» (S. 440). «Unsere genuin sozialwissenschaftliche Prozeßkonzeption steht in krassem Widerspruch zu Prozeßtheorien, die ideologisch von einer vermeintlichen Naturwüchsigkeit ausgehen.»(S. 445) Darin ist die Bemühung um eine «heuristisch orientierte und am Suchen, Finden und Entdecken sowie an der Herstellung der bestmöglichen Veränderungsbedingungen für den Patienten ausgerichtete Technik impliziert» (S. 446).

Im letzten Kapitel beschäftigen sich die Autoren mit dem Verhältnis von Theorie und Praxis und hinterfragen das Freudsche Junktim zwischen Heilen und Forschen. Sie unterscheiden innerhalb des psychoanalytischen Wissens deskriptives und klassifikatorisches Wissen (was ist?), Bedingungswissen (warum ist es so?) und Therapie- und Änderungswissen.
Durch letzteres wird die klinisch-psychoanalytische Forschung und Behandlung weitgehend geregelt (S. 456). «Auch die psychoanalytische Therapie lebt, wenn auch in besonders ausgefeilter Weise, von allgemeinen Ingredienzien hilfreicher Therapie, wie es Luborsky (1984) an der helping alliance> empirisch aufgewiesen hat. Darüber hinaus weisen psychoanalytische Therapieformen Spezifika auf, die sie mehr oder weniger deutlich von anderen Therapieansätzen unterscheiden. Wir neigen zu der Ansicht, daß die exakte Erforschung der Veränderungsprozesse in der psychoanalytischen Therapie am Anfang steht und eine Vielzahl von detaillierten Studien ... erst noch durchgeführt werden muß» (S. 467). Der klinisch-systematischen Untersuchung des Behandlungsmaterials im Sinne einer technologischen Forschung, d. h. Forschung zur psychoanalytischen Technik und Technologie, fühlen sich die Autoren verpflichtet.

Insgesamt stellt dieser erste Band des Lehrbuchs von Thomä/ Kächele eine äußerst lohnende und materialreiche Auseinandersetzung mit essentiellen Fragen moderner psychoanalytischer Therapie dar. Für mich persönlich besonders erhellend war die detaillierte Darstellung der vielfältigen Auswirkungen der triebökonomischen Metapsychologie Freuds auf die Praxis der Psychoanalyse. Gerade in entschiedener Auseinandersetzung mit diesem Modell und in der angstfreien Ablösung von doktrinärer Bewahrung eines «klassischen» Lehrgebäudes ohne Aufgabe der «essentials» wird der Reichtum psychoanalytischen Wissens neu angeeignet und ein Brückenschlag zu anderen Theorieansätzen dort versucht, wo er möglich scheint. Dies alles macht das Buch m. E. auch für Leser interessant, die als Therapeuten oder Forscher außerhalb der «psychoanalytischen Gemeinde» tätig sind.

R. Neumeier, Mannheim 